\title{
A high-resolution map of human evolutionary constraint using 29 mammals
}

\author{
Kerstin Lindblad-Toh ${ }^{1,2}$, Manuel Garber ${ }^{1 *}$, Or Zuk ${ }^{1 *}$, Michael F. Lin ${ }^{1,3 *}$, Brian J. Parker ${ }^{4 *}$, Stefan Washietl ${ }^{3 *}$, \\ Pouya Kheradpour ${ }^{1,3 *}$, Jason Ernst ${ }^{1,3 *}$, Gregory Jordan ${ }^{5 *}$, Evan Mauceli ${ }^{1 *}$, Lucas D. Ward ${ }^{1,3 *}$, Craig B. Lowe ${ }^{6,7,8 *}$, \\ Alisha K. Holloway ${ }^{9}$, , Michele Clamp ${ }^{1,10 *}$, Sante Gnerre ${ }^{1 *}$, Jessica Alföldi ${ }^{1}$, Kathryn Beal ${ }^{5}$, Jean Chang ${ }^{1}$, Hiram Clawson ${ }^{6}$, \\ James Cuff $^{11}$, Federica Di Palma ${ }^{1}$, Stephen Fitzgerald ${ }^{5}$, Paul Flicek ${ }^{5}$, Mitchell Guttman ${ }^{1}$, Melissa J. Hubisz ${ }^{12}$, David B. Jaffe ${ }^{1}$, \\ Irwin Jungreis ${ }^{3}$, W. James Kent ${ }^{9}$, Dennis Kostka ${ }^{9}$, Marcia Lara ${ }^{1}$, Andre L. Martins ${ }^{12}$, Tim Massingham ${ }^{5}$, Ida Moltke ${ }^{4}$, \\ Brian J. Raney ${ }^{6}$, Matthew D. Rasmussen ${ }^{3}$, Jim Robinson ${ }^{1}$, Alexander Stark ${ }^{13}$, Albert J. Vilella ${ }^{5}$, Jiayu Wen ${ }^{4}$, Xiaohui Xie ${ }^{1}$, \\ Michael C. Zody ${ }^{1}$, Broad Institute Sequencing Platform and Whole Genome Assembly Team†, Kim C. Worley ${ }^{14}$, Christie L. Kovar ${ }^{14}$, \\ Donna M. Muzny ${ }^{14}$, Richard A. Gibbs ${ }^{14}$, Baylor College of Medicine Human Genome Sequencing Center Sequencing Team $\dagger$, \\ Wesley C. Warren ${ }^{15}$, Elaine R. Mardis ${ }^{15}$, George M. Weinstock ${ }^{14,15}$, Richard K. Wilson ${ }^{15}$, Genome Institute at Washington \\ University†, Ewan Birney ${ }^{5}$, Elliott H. Margulies ${ }^{16}$, Javier Herrero ${ }^{5}$, Eric D. Green ${ }^{17}$, David Haussler ${ }^{6,8}$, Adam Siepel ${ }^{12}$, \\ Nick Goldman ${ }^{5}$, Katherine S. Pollard ${ }^{9,18}$, Jakob S. Pedersen ${ }^{4,19}$, Eric S. Lander ${ }^{1}$ \& Manolis Kellis ${ }^{1,3}$
}

The comparison of related genomes has emerged as a powerful lens for genome interpretation. Here we report the sequencing and comparative analysis of 29 eutherian genomes. We confirm that at least $5.5 \%$ of the human genome has undergone purifying selection, and locate constrained elements covering $\sim 4.2 \%$ of the genome. We use evolutionary signatures and comparisons with experimental data sets to suggest candidate functions for $\sim 60 \%$ of constrained bases. These elements reveal a small number of new coding exons, candidate stop codon readthrough events and over 10,000 regions of overlapping synonymous constraint within protein-coding exons. We find 220 candidate RNA structural families, and nearly a million elements overlapping potential promoter, enhancer and insulator regions. We report specific amino acid residues that have undergone positive selection, 280,000 non-coding elements exapted from mobile elements and more than 1,000 primate- and human-accelerated elements. Overlap with disease-associated variants indicates that our findings will be relevant for studies of human biology, health and disease.

A key goal in understanding the human genome is to discover and interpret all functional elements encoded within its sequence. Although only $\sim 1.5 \%$ of the human genome encodes protein sequence ${ }^{1}$, comparative analysis with the mouse ${ }^{2}$, rat $^{3}$ and $\mathrm{dog}^{4}$ genomes showed that at least $5 \%$ is under purifying selection and thus probably functional, of which $\sim 3.5 \%$ consists of non-coding elements with probable regulatory roles. Detecting and interpreting these elements is particularly relevant to medicine, as loci identified in genome-wide association studies (GWAS) frequently lie in non-coding sequence ${ }^{5}$.

Although initial comparative mammalian studies could estimate the overall proportion of the genome under evolutionary constraint, they had little power to detect most of the constrained elementsespecially the smaller ones. Thus, they focused only on the top $5 \%$ of constrained sequence, corresponding to less than $\sim 0.2 \%$ of the genome $e^{4,6}$. In 2005 , we began an effort to generate sequence from a large collection of mammalian genomes with the specific goal of identifying and interpreting functional elements in the human genome on the basis of their evolutionary signatures ${ }^{7,8}$. Here we report our results to systematically characterize mammalian constraint using 29 eutherian (placental) genomes. We identify $4.2 \%$ of the human genome as constrained and ascribe potential function to $\sim 60 \%$ of these bases using diverse lines of evidence for protein-coding, RNA, regulatory and chromatin roles, and we present evidence of exaptation and accelerated evolution. All data sets described here are publicly available in a comprehensive data set at the Broad Institute and University of California, Santa Cruz (UCSC).

\section{Sequencing, assembly and alignment}

We generated genome sequence assemblies for 29 mammalian species selected to achieve maximum divergence across the four major mammalian clades (Fig. 1a and Supplementary Text 1 and Supplementary Table 1). For nine species, we used genome assemblies based on $\sim 7$ fold coverage shotgun sequence, and for 20 species we generated $\sim 2$ fold coverage $(2 \times)$, to maximize the number of species sequenced

${ }^{1}$ Broad Institute of Harvard and Massachusetts Institute of Technology (MIT), 7 Cambridge Center, Cambridge, Massachusetts 02142, USA. ${ }^{2}$ Science for Life Laboratory, Department of Medical

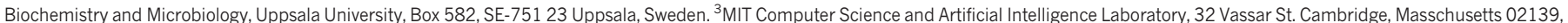

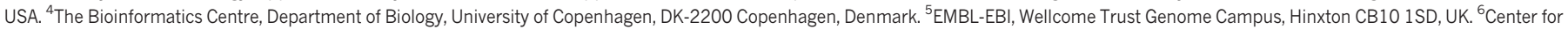

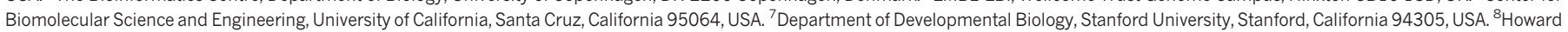

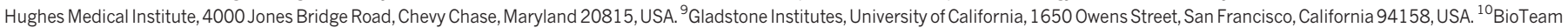
Inc, 7 Derosier Drive, Middleton, Massachusetts 01949, USA. ${ }^{11}$ Research Computing, Division of Science, Faculty of Arts and Sciences, Harvard University, Cambridge, Massachusetts 02138, USA.

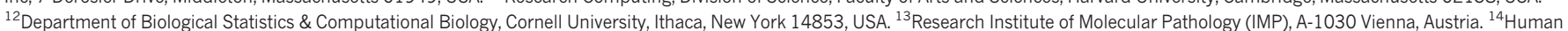

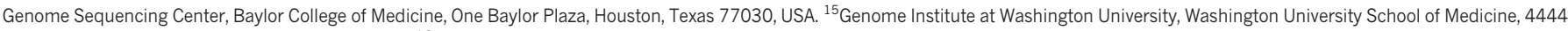

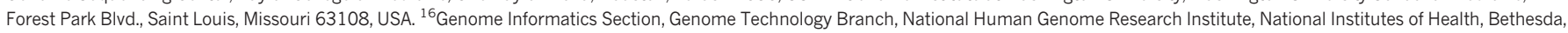

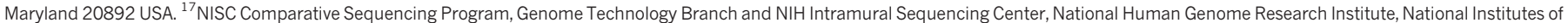

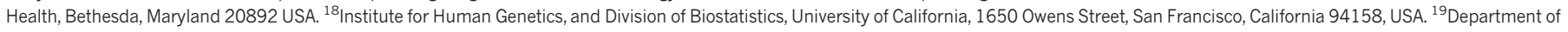
Molecular Medicine (MOMA), Aarhus University Hospital, Skejby, DK-8200 Aarhus N, Denmark. 
a

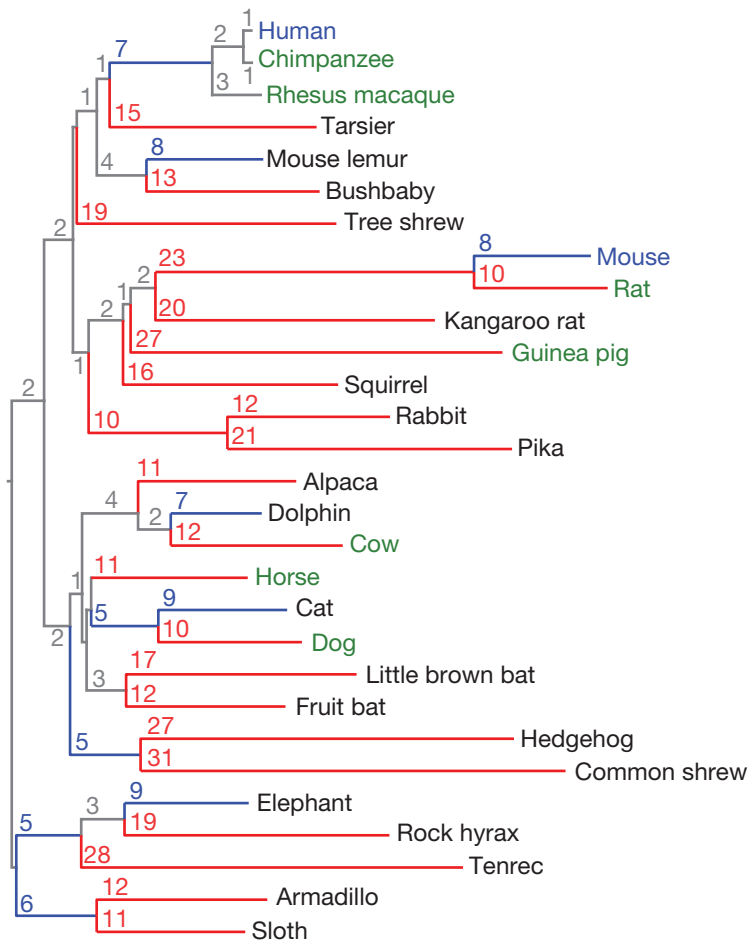

b

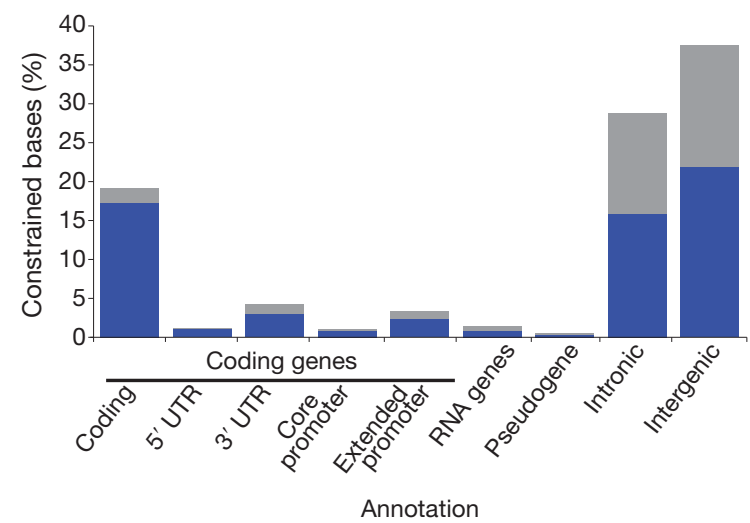

Figure $1 \mid$ Phylogeny and constrained elements from the 29 eutherian mammalian genome sequences. a, A phylogenetic tree of all 29 mammals used in this analysis based on the substitution rates in the MultiZ alignments. Organisms with finished genome sequences are indicated in blue, high quality drafts in green and $2 \times$ assemblies in black. Substitutions per $100 \mathrm{bp}$ are given for each branch; branches with $\geq 10$ substitutions are coloured red, blue indicates $<10$ substitutions. b, At 10\% FDR, 3.6 million constrained elements can be detected encompassing $4.2 \%$ of the genome, including a substantial fraction of newly detected bases (blue) compared to the union of the HMRD 50 $\mathrm{bp}+$ Siepel vertebrate elements ${ }^{17}$ (see Supplementary Fig. $4 \mathrm{~b}$ for comparison to HMRD elements only). The largest fraction of constraint can be seen in coding exons, introns and intergenic regions. For unique counts, the analysis was performed hierarchically: coding exons, 5' UTRs, 3' UTRs, promoters, pseudogenes, non-coding RNAs, introns, intergenic. The constrained bases are particularly enriched in coding transcripts and their promoters (Supplementary Fig. 4c).

with available resources on capillary machines. Twenty genomes are first reported here, and nine were previously described (see Supplementary Information).

The power to detect constrained elements depends largely on the total branch length of the phylogenetic tree connecting the species 9 . The 29 mammals correspond to a total effective branch length of $\sim 4.5$ substitutions per site, compared to $\sim 0.68$ for the human-mouserat-dog comparison (HMRD), and thus should offer greater power to detect evolutionary constraint: the probability that a genomic sequence not under purifying selection will remain fixed across all 29 species is $P_{1}<0.02$ for single bases and $P_{12}<10^{-25}$ for 12-nucleotide sequences, compared to $P_{1} \sim 0.50$ and $P_{12} \sim 10^{-3}$ for HMRD.

For mammals for which we generated $2 \times$ coverage, our assisted assembly approach ${ }^{10}$ resulted in a typical contig size $\mathrm{N} 50_{\mathrm{C}}$ of $2.8 \mathrm{~kb}$ and a typical scaffold size $\mathrm{N}^{2} 0_{\mathrm{S}}$ of $51.8 \mathrm{~kb}$ (Supplementary Text 2 and Supplementary Table 1) and high sequence accuracy (96\% of bases had quality score Q20, corresponding to a $<1 \%$ error rate $)^{11}$. Compared to high-quality sequence across the $30 \mathrm{Mb}$ of the ENCODE pilot project ${ }^{12}$, we estimated average error rates of $1-3$ miscalled bases per kilobase ${ }^{11}$, which is $\sim 50$-fold lower than the typical nucleotide sequence difference between the species, enabling high-confidence detection of evolutionary constraint (Supplementary Text 3).

We based our analysis on whole-genome alignments by MultiZ (Supplementary Text 4). The average number of aligned species was 20.9 at protein-coding positions in the human genome and 23.9 at the top 5\% HMRD-conserved non-coding positions, with an average branch length of 4.3 substitutions per base in these regions (Supplementary Figs 1 and 2). In contrast, whole-genome average alignment depth is only 17.1 species with 2.9 substitutions per site, probably due to large deletions in non-functional regions ${ }^{4}$. The depth at ancestral repeats is 11.4 (Supplementary Fig. 1a), consistent with repeats being largely non-functional ${ }^{2,4}$.

\section{Detection of constrained sequence}

Our analysis did not substantially change the estimate of the proportion of genome under selection. By comparing genome-wide conservation to that of ancestral repeats, we estimated the overall fraction of the genome under evolutionary constraint to be $5.36 \%$ at 50 -bp windows (5.44\% at 12 -bp windows), using the SiPhy- $\omega$ statistic ${ }^{13}$, a measure of overall substitution rate (Supplementary Fig. 3), consistent with previous similar estimates ${ }^{2,4,14}$. However, alternative methods ${ }^{15,16}$ and different ways of correcting for the varying alignment depths give higher estimates (see Supplementary Text 5 for details).

The additional species had a marked effect on our ability to identify the specific elements under constraint. With 29 mammals, we pinpoint 3.6 million elements spanning $4.2 \%$ of the genome, at a finer resolution of 12 bp (Fig. 1b and Supplementary Text 6, Supplementary Fig. 4, Supplementary Tables 2 and 3), compared to $<0.1 \%$ of the genome for HMRD 12-bp elements and 2.0\% for HMRD 50-bp elements ${ }^{4}$. Elements previously detected using five vertebrates ${ }^{17}$ also detect a larger fraction of the genome $(\sim 4.1 \%)$, but only cover $45 \%$ of the mammalian elements detected here, suggesting that a large fraction of our elements are mammalian specific. The mean element size (36 bp) is considerably shorter than both previously detected HMRD elements (123 bp) and five-vertebrate elements $(104 \mathrm{bp})^{17}$. For example, it is now possible to detect individual binding sites for the neuron-restrictive silencer factor (NRSF) in the promoter of the NPAS4 gene, which are beyond detection power in previous data sets (Fig. 2 and Supplementary Fig. 5). We found a similar regional distribution of 12-bp elements (including the 2.6 million newly detected constrained elements) to previously detected HMRD elements $(r=0.94$, Supplementary Fig. 6). Similar results were obtained with the PhastCons ${ }^{17}$ statistic (see Supplementary Text 6).

Using a new method, SiPhy- $\pi$, sensitive not just to the substitution rate but also to biases in the substitution pattern (for example, positions free to mutate between G and T only, Supplementary Fig. 7), we detected an additional $1.3 \%$ of the human genome in constrained elements (see Supplementary Tables 2 and 3). Most of the newly detected constrained nucleotides extend elements found by ratebased methods, but $22 \%$ of nucleotides lie in new elements (average length $17 \mathrm{bp}$ ) and are enriched in non-coding regions. 
a

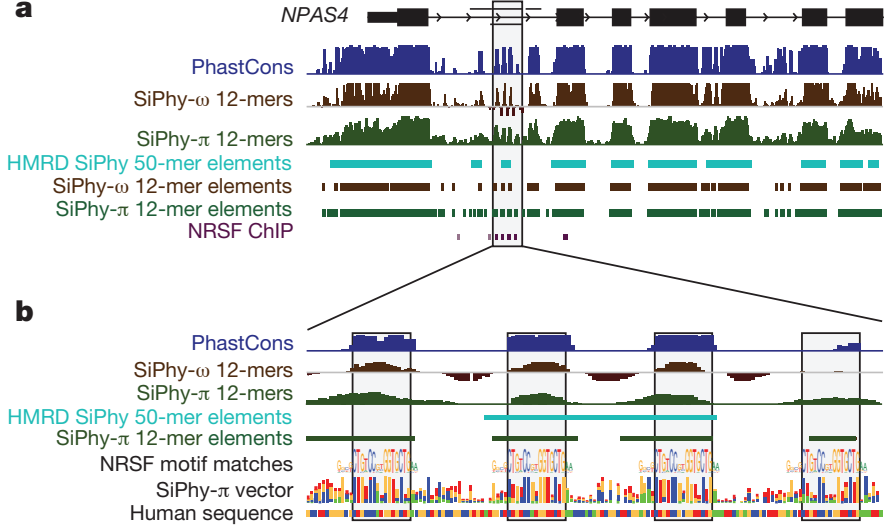

Figure $2 \mid$ Identification of four NRSF-binding sites in NPAS4. a, The neurological gene NPAS4 has many constrained elements overlapping introns and the upstream intergenic region. The grey shaded box contained only one constrained element using HMRD, whereas analysis of 29 mammalian sequences reveals four smaller elements. $\mathbf{b}$, These four constrained elements in the first intron correspond to binding sites for the NRSF transcription factor, known to regulate neuronal lineages.

\section{Constraint within the human population}

We observed that the evolutionary constraint acting on the 29 mammals is correlated with constraint within the human population, as assessed from human polymorphism data (Supplementary Text 7) and consistent with previous studies ${ }^{18}$. Mammalian constrained elements show a depletion in single-nucleotide polymorphisms $(\mathrm{SNPs})^{19}$, and more constrained elements show even greater depletion. For example, in the top $1 \%$ most strongly conserved non-coding regions, SNPs occur at a 1.9-fold lower rate than the genome average, and the derived alleles have a lower frequency, consistent with purifying selection at many of these sites in the human population.

Moreover, at positions with biased substitution patterns across mammals, the observed human SNPs show a similar bias to the one observed across mammals (Supplementary Fig. 7). Thus, not only are constrained regions less likely to exhibit polymorphism in humans, but when such polymorphisms are observed, the derived alleles in humans tend to match the alleles present in non-human mammals, indicating a preference for the same alleles across both mammalian and human evolution.

\section{Functional annotation of constraint}

We first studied the overlap of the 3.6 million evolutionarily constrained elements $\left(\omega<0.8\right.$ and $\left.P<10^{-15}\right)$ with known gene annotations (Fig. 1b). Roughly $30 \%$ of constrained elements were associated with protein-coding transcripts: $\sim 25.3 \%$ overlap mature messenger RNAs (including $19.6 \%$ in coding exons, $1.2 \%$ in $5^{\prime}$ untranslated regions ( $5^{\prime}$ UTRs) and $4.4 \%$ in $3^{\prime}$ UTRs), and an additional $4.4 \%$ reside within $2 \mathrm{~kb}$ of transcriptional start sites (1.2\% reside within 200 bases).

The majority of constrained elements, however, reside in intronic and intergenic regions ( $29.7 \%$ and $38.6 \%$, respectively). To study their biological roles and provide potential starting points to understand these large and mostly uncharted territories, we next studied their overlap with evolutionary signatures ${ }^{7,8,20,21}$ characteristic of specific types of features and a growing collection of public large-scale experimental data.

\section{Protein-coding genes and exons}

Despite intense efforts to annotate protein-coding genes over the past

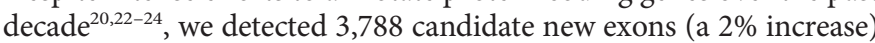
using evolutionary signatures characteristic of protein-coding exons ${ }^{25}$. Of these, 54\% reside outside transcripts of protein-coding genes, 19\% within introns, and $13 \%$ in UTRs of known coding genes (Supplementary Text 8 , Supplementary Tables 4 and 5). Our methods recovered
$92 \%$ of known coding exons that were larger than 10 codons and fall in syntenic regions, the remainder showing non-consensus splice sites, unusual features, or poor conservation.

The majority of new exon candidates $(>58 \%)$ are supported by evidence of transcription measured in 16 human tissues ${ }^{26}$ (Supplementary Fig. 8a) or similarity to known Pfam protein domains. Thirty-one per cent of intronic and $13 \%$ of intergenic predictions extend known transcripts, and $5 \%$ and $11 \%$ respectively reside in new transcript models. The newly detected exons are more tissue specific than known exons (mean of 3 tissues versus 12) and are expressed at fivefold lower levels. Directed experiments and manual curation will be required to complete the annotation of the few hundred protein-coding genes that probably remain unannotated ${ }^{27}$.

We found apparent stop codon readthrough ${ }^{28}$ of four genes based on continued protein-coding constraint after an initial conserved stop $\operatorname{codon}^{29}$ and until a subsequent stop codon (Supplementary Text 9 and Supplementary Fig. 8b). Readthrough in SACM1L could be triggered by an 80 -base conserved RNA stem loop predicted by $\mathrm{RNAz}^{30}$, lying four bases downstream of the readthrough stop codon.

We also detected coding regions with a very low synonymous substitution rate, indicating additional sequence constraints beyond the amino acid level (Supplementary Text 9). We found $>10,000$ such synonymous constraint elements (SCEs) in more than one-quarter of all human genes ${ }^{31}$. Initial analysis indicates potential roles in splicing regulation (34\% span an exon-exon junction), A-to-I editing, microRNA (miRNA) targeting and developmental regulation. HOX genes contain several top candidates (Fig. 3a), including two previously validated developmental enhancers ${ }^{32,33}$.

\section{RNA structures and families of structural elements}

We next used evolutionary signatures characteristic of conserved RNA secondary structures ${ }^{34}$ to reveal 37,381 candidate structural elements (Supplementary Text 10 and Supplementary Fig. 9a), covering $\sim 1 \%$ of constrained regions. For example, the XIST large intergenic non-coding RNA (lincRNA), known to bind chromatin and enable $\mathrm{X}$-chromosome inactivation ${ }^{35}$, contains a newly predicted structure in its $3^{\prime}$ end (Supplementary Fig. 9b, f)-distinct from other known structures ${ }^{36}$ - which seems to be the source of chromatin-associated short RNAs ${ }^{37}$.

Sequence- and structure-based clustering of predictions outside protein-coding exons revealed 1,192 novel families of structural RNAs (Supplementary Text 10). We focused on a high-scoring subset consisting of 220 families with 725 instances, which also showed the highest thermodynamic stability ${ }^{30}$ (Supplementary Figs 9a and 10), DNase hypersensitivity, expression pattern correlation across tissues and intergenic expression enrichment (Supplementary Fig. 9a). We also expanded both known and novel families by including additional members detected by homology to existing members.

Noteworthy examples include: a glycyl-tRNA family, including a new member in $P O P 1$, involved in tRNA maturation and probably involved in feedback regulation of $P O P 1$; three intronic families of long hairpins in ion-channel genes known to undergo A-to-I RNA editing and possibly involved in regulation of the editing event; an additional member of a family of 5' UTR hairpins overlapping the start codon of collagen genes and potential new miRNA genes that extend existing families ${ }^{37}$.

Two of the largest novel families consist of short AU-rich hairpins of 6-7 bp that share the same strong consensus motif in their stem. These occur in the 3' UTRs of genes in several inflammatory response pathways, the post-transcriptional regulation of which often involves AUrich elements (AREs). Indeed, two homologous hairpins in TNF and CSF3 correspond to known mRNA-destabilization elements, suggesting roles in mRNA stability for the two families ${ }^{37}$.

Lastly, a family of six conserved hairpin structures (Supplementary Fig. 9g) was found in the $3^{\prime}$ UTR of the MAT2A gene ${ }^{37}$, which is involved in the synthesis of $S$-adenosylmethionine (SAM), the primary 


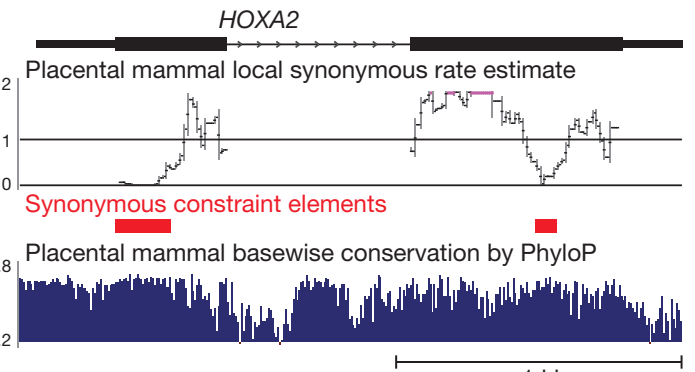

$1 \mathrm{~kb}$

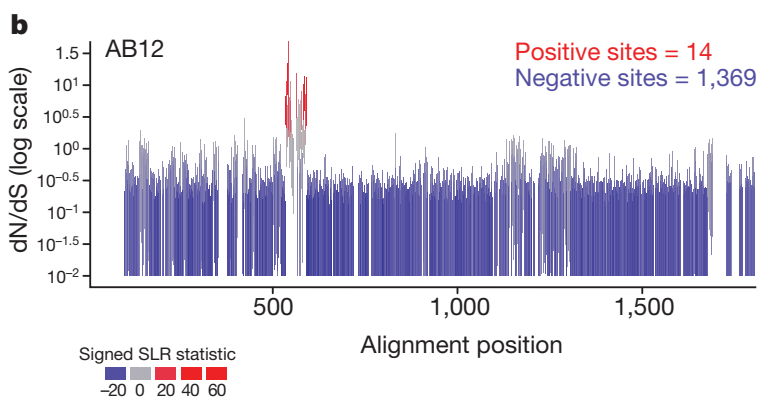

Figure 3 Examination of evolutionary signatures identifies SCEs and evidence of positive selection. a, Two regions within the HOXA2 open reading frame are identified as SCEs (red), corresponding to overlapping functional elements within coding regions. Note that the synonymous rate reductions are not obvious from the base-wise conservation measure (in blue). Both elements have been characterized as enhancers driving HOXA2 expression in distinct segments of the developing mouse hindbrain. The element in the first exon encodes Hox-Pbx-binding sites and drives expression in rhombomere 4 (ref. 33 ), whereas the element in the second exon contains Sox-binding sites and drives expression in rhombomere 2 (ref. 32). Synonymous constraint elements are also found in most other HOX genes, and up to a quarter of all genes. b, Although $\sim 85 \%$ of genes show only negative (purifying) selection and $9 \%$ of genes show uniform positive selection, the remaining $6 \%$ of genes, including $A B I 2$, show only localized regions of positively selected sites. Each vertical bar covers the estimated $95 \%$ confidence interval for $\mathrm{dN} / \mathrm{dS}$ at that site (with values of 0 truncated to 0.01 to accommodate the log scaling), and bars are coloured according to a signed version of the SLR statistic for non-neutral evolution: blue for sites under purifying selection, grey for neutral sites and red for sites under positive selection.

methyl donor in human cells. All six hairpins consist of a 12-18-bp stem and a 14-bp loop region with a deeply conserved sequence motif (Supplementary Fig. 9e), and may be involved in sensing SAM concentrations, which are known to affect MAT2A mRNA stability ${ }^{38}$.

\section{Conservation patterns in promoters}

As different types of conservation in promoters may imply distinct biological functions ${ }^{39}$, we classified the patterns of conservation within core promoters into three categories: (1) those with uniformly 'high' constraint (7,635 genes, 13,996 transcripts); (2) uniformly 'low' constraint (2,879 genes, 4,135 transcripts); and (3) 'intermittent' constraint, consisting of alternating peaks and troughs of conservation (14,271 genes and 29,814 transcripts) (Supplementary Fig. 11a). High and intermittent constraint promoters are both associated with $\mathrm{CpG}$ islands $(\sim 66 \%)$, whereas low constraint promoters have significantly lower overlap $(\sim 41 \%)$, and all three classes show similar overlap with functional TATA boxes (2-3\%, see Supplementary Text 11).

These groups show distinct Gene Ontology enrichments (Supplementary Fig. 11b), with high-constraint promoters involved in development $\left(P\right.$ with Bonferroni correction $\left(P_{\text {Bonf }}\right)<10^{-30}$ ), intermittent constraint in basic cellular functions $\left(P_{\text {Bonf }}<5 \times 10^{-4}\right)$, and low-constraint promoters in immunity, reproduction and perception, functions expected to be under positive selection and lineage-specific adaptation $^{2}$.
High constraint may reflect cooperative binding of many densely binding factors, as previously suggested for developmental genes ${ }^{6}$. Intermittent constraint promoters, the peak-spacing distribution of which was suggestive of the periodicity of the DNA helix turns, may reflect loosely interacting factors (Supplementary Fig. 11c, d). Low constraint may reflect rapid motif turnover, under neutral drift or positive selection.

\section{Identifying specific instances of regulatory motifs}

Data from just four species (HMRD) was sufficient to create a catalogue of known and novel motifs with many conserved instances across the genome ${ }^{21}$. The power to discover such motifs was high, because one can aggregate data across hundreds of motif instances. Not surprisingly, the additional genomes therefore had little effect on the ability to discover new motifs (known motifs showed 99\% correlation in genome-wide motif conservation scores, Supplementary Figs 12 and 13).

In contrast, the 29 mammalian genomes markedly improved our ability to detect individual motif instances, making it possible to predict specific target sites for 688 regulatory motifs corresponding to 345 transcription factors (Supplementary Fig. 14). We chose to identify motif instances at a false discovery rate (FDR) of $60 \%$, representing a reasonable compromise between specificity and sensitivity given the available discovery power (Supplementary Text 12), and matching the experimental specificity of chromatin immunoprecipitation (ChIP) experiments for identifying biologically significant targets ${ }^{40}$. Higher levels of stringency could be obtained by sequencing additional species.

We identified 2.7 million conserved instances (Supplementary Table 6), enabling the construction of a regulatory network linking 375 motifs to predicted targets, with a median of 21 predicted regulators per target gene (25th percentile, 10; 75th percentile, 39). The number of target sites (average, 4,277; 25 th percentile, 1,407; 75th percentile, 10,782) are comparable to those found in ChIP experiments, and have the advantage that they are detected at nucleotide resolution, enabling us to use them to interpret disease-associated variants for potential regulatory functions. However, some motifs never reached high confidence values, and others did so at very few instances.

The motif-based targets show strong agreement with experimentally defined binding sites from ChIP experiments (Supplementary Table 7). For long and distinct motifs, such as CTCF and NRSF, the fraction of instances overlapping experimentally observed binding matches the fraction predicted by the confidence score (for example, at $80 \%$ confidence $70 \%$ of NRSF motif instances overlapped bound sites, and at $\sim 50 \%$ confidence $40 \%$ overlapped), despite potential confounding aspects such as condition-specific binding, overlapping motifs between factors, or non-specific binding. Moreover, increasing confidence levels showed increasing overlap with experimental binding (Supplementary Figs 14-16). For example, YY1 enrichment for bound sites increased from 42 -fold to 168 -fold by focusing on conserved instances. Lastly, combining motif conservation and experimental binding led to increased enrichment for candidate tissue-specific enhancers, suggesting that the two provide complementary information. Within bound regions, the evolutionary signal reveals specific motif instances with high precision (for example, Figs 2 and 4 and Supplementary Fig. 17).

\section{Chromatin signatures}

To suggest potential functions for the $\sim 68 \%$ of 'unexplained' constrained elements outside coding regions, UTRs or proximal promoters, we used chromatin state maps from CD4 T cells ${ }^{41}$ (Supplementary Fig. 18) and nine diverse cell types ${ }^{42}$ (Supplementary Text 13 and Supplementary Fig. 19). In $\mathrm{T}$ cells, constrained elements were most enriched for promoter-associated states (up to fivefold), an insulator state and a specific repressed state (2.2-fold), and numerous enhancer 

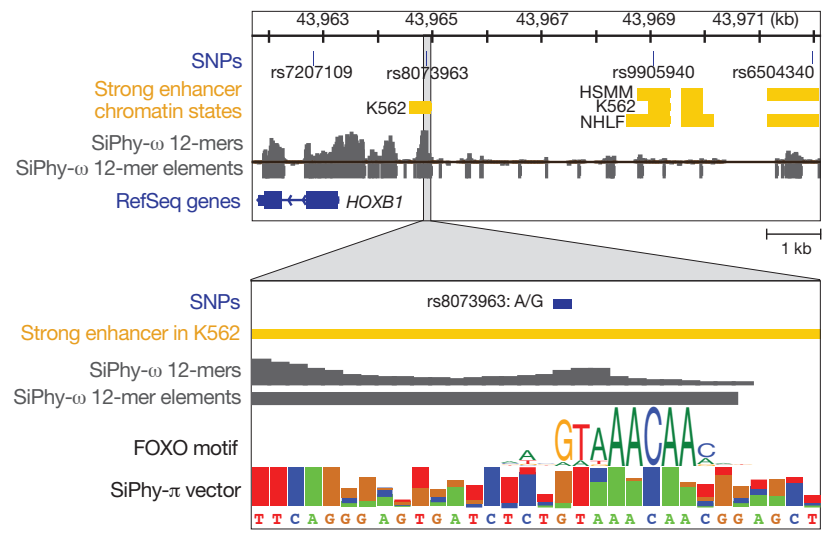

Figure $4 \mid$ Using constraint to identify candidate mutations. Conservation can help us resolve amid multiple SNPs the ones that disrupt conserved functional elements and are likely to have regulatory roles. In this example, a SNP (rs6504340) associated with tooth development is strongly linked to a conserved intergenic SNP, rs8073963, $7.1 \mathrm{~kb}$ away, which disrupts a deeply conserved Forkhead-family motif in a strong enhancer. Although the SNPs shown here stem from GWAS on HapMap data, the same principle should be applicable to associated variants detected by resequencing the region of interest.

states (1.5-2-fold), together covering 7.1\% of the unexplained elements at 2.1-fold enrichment. In the nine cell types, enriched promoter, enhancer and insulator states cover $36 \%$ of unexplained elements at $\sim 1.75$-fold enrichment, with locations active in multiple cell types showing even stronger enrichment (Supplementary Fig. 20).

Overall, chromatin states indicate possible functions (at 1.74-fold enrichment) for $37.5 \%(N=987,985)$ of unexplained conserved elements (27\% of all conserved elements), suggesting meaningful association for at least $16 \%$ of unexplained constrained bases. Although current experiments only provide nucleosome-scale ( $\sim 200$-bp) resolution, we expect higher-resolution experimental assays that more precisely pinpoint regulatory regions to show further increases in enrichment. The increased overlap observed with additional cell types suggests that new cell types will help elucidate additional elements. Of course, further experimental tests will be required to validate the predicted functional roles.

\section{Accounting for constrained elements}

Overall, $\sim 30 \%$ of constrained elements overlap were associated with protein-coding transcripts, $\sim 27 \%$ overlap specific enriched chromatin states, $\sim 1.5 \%$ novel RNA structures, and $\sim 3 \%$ conserved regulatory motif instances (Supplementary Text 13, 14). Together, $\sim 60 \%$ of constrained elements overlap one of these features, with enrichments ranging from 1.75-fold for chromatin states (compared to unannotated regions) up to 17 -fold for protein-coding exons (compared to the whole genome).

\section{Implications for interpreting disease-associated variants}

In the non-protein-coding genome, SNPs associated with human diseases in genome-wide association studies are 1.37-fold enriched for constrained regions, relative to HapMap SNPs (Supplementary Text 15 and Supplementary Table 8). This is notable because only a small proportion of the associated SNPs are likely to be causative, whereas the rest are merely in linkage disequilibrium with causative variants.

Accordingly, constrained elements should be valuable in focusing the search for causative variants among multiple variants in linkage disequilibrium. For example, in an intergenic region between $H O X B 1$ and HOXB2 associated with tooth development phenotypes ${ }^{43}$, the reported SNP (rs6504340) is not conserved, but a linked SNP (rs8073963) sits in a constrained element $7.1 \mathrm{~kb}$ away. Moreover, rs8073963 disrupts a deeply conserved FOXO2 motif instance within a predicted enhancer (Fig. 4), making it a candidate mutation for further follow-up. Similar examples of candidate causal variants are found for diverse phenotypes such as height or multiple sclerosis, and similar analyses could be applied to case-control resequencing data.

\section{Evolution of constrained elements}

We next sought to identify signatures of positive selection that may accompany functional adaptations of different species to diverse environments and new ecosystems.

\section{Codon-specific selection}

We used the ratio $\mathrm{dN} / \mathrm{dS}$ of non-synonymous to synonymous codon substitutions as evidence of positive selection $(>1)$ or negative selection $(<1)$. Although $\mathrm{dN} / \mathrm{dS}$ is typically calculated for whole genes, the additional mammals sequenced enabled analysis at the codon level: simulations predicted a 250 -fold gain in sensitivity compared to HMRD, identifying $53 \%$ of positive sites at 5\% FDR (Supplementary Text 16).

Applying this test to 6.05 million codons in 12,871 gene trees, we found evidence of strong purifying selection $(\mathrm{dN} / \mathrm{dS}<0.5)$ for $84.2 \%$ of codons and positive selection ( $\mathrm{dN} / \mathrm{dS}>1.5)$ for $2.4 \%$ of codons (with $94.1 \%$ of sites $<1$ and $5.9 \%>1$; Supplementary Table 9). At 5\% FDR, we found 15,383 positively selected sites in 4,431 proteins. The genes fall into three classes based on the distribution of selective constraint: $84.8 \%$ of genes show uniformly high purifying selection, $8.9 \%$ show distributed positive selection across their length and $6.3 \%$ show localized positive selection concentrated in small clusters (Fig. 3b and Supplementary Fig. 21, Supplementary Tables 10 and 11).

Genes with distributed positive selection were enriched in such functional categories as immune response $\left(P_{\text {Bonf }}<10^{-16}\right)$ and taste perception $\left(P_{\text {Bonf }}<10^{-10}\right)$, which are known to evolve rapidly, but also in some unexpected functions such as meiotic chromosome segregation $\left(P_{\mathrm{Bonf}}<10^{-23}\right)$ and DNA-dependent regulation of transcription $\left(P_{\text {Bonf }}<10^{-19}\right.$; Supplementary Table 12$)$. Localized positive selection was enriched in core biochemical processes, including microtubule-based movement $\left(P_{\mathrm{Bonf}}<10^{-10}\right)$, DNA topological change $\left(P_{\text {Bonf }}<10^{-4}\right)$ and telomere maintenance $\left(P_{\text {Bonf }}<7 \times 10^{-3}\right)$, suggesting adaptation at important functional sites.

Focusing on 451 unique Pfam protein-domain annotations, we found abundant purifying selection, with 225 domains showing purifying selection for $>75 \%$ of their sites, and 447 domains showing negative selection for $>50 \%$ of their sites (Supplementary Table 13). Domains with substantial fractions of positively selected sites include CRAL/TRIO involved in retinal binding $(2.6 \%)$, proteinase-inhibitorcystatin involved in bone remodelling $(2.2 \%)$ and the secretion-related EMP24/GOLD/P24 family (1.6\%).

\section{Exaptation of mobile elements}

Mobile elements provide an elegant mechanism for distributing a common sequence across the genome, which can then be retained in locations where it confers advantageous regulatory functions to the host-a process termed exaptation. Our data revealed $>280,000$ mobile element exaptations common to mammalian genomes covering $\sim 7 \mathrm{Mb}$ (Supplementary Text 17), a considerable expansion from the $\sim 10,000$ previously recognized cases ${ }^{44}$. Of the $\sim 1.1$ million constrained elements that arose during the 90 million years between the divergence from marsupials and the eutherian radiation, we can trace $>19 \%$ to mobile element exaptations. Often only a small fraction (median $\sim 11 \%$ ) of each mobile element is constrained, in some cases matching known regulatory motifs. Recent exaptations are generally found near ancestral regulatory elements, except in gene deserts, which are abundant in ancestral elements but show few recent exaptations $\left(P<10^{-300}\right.$, Supplementary Fig. 22).

\section{Accelerated evolution in the primate lineage}

Lineage-specific rapid evolution in ancestrally constrained elements previously revealed human positive selection associated with brain 
and limb development ${ }^{45}$. Applying this signature to the human and primate lineages, we identified 563 human-accelerated regions (HARs) and 577 primate-accelerated regions (PARs) at FDR $<10 \%$ (Supplementary Text 18, Supplementary Tables 14 and 15), significantly expanding the 202 previously known HARs ${ }^{46}$. Fifty-four HARs (9.4\%) and 49 PARs (8.5\%) overlap enhancer-associated chromatin marks and experimentally validated enhancers (Supplementary Text 18). Substitution patterns in HARs suggest that GC-biased gene conversion (BGC) is not responsible for the accelerated evolution in the vast majority of these regions ( $\sim 15 \%$ show evidence of BGC).

Genes harbouring or neighbouring HARs and PARs are enriched for extracellular signalling, receptor activity, immunity, axon guidance, cartilage development and embryonic pattern specification (Supplementary Fig. 23). For example, the FGF13 locus associated with an X-linked form of mental retardation contains four HARs near the 5 ' ends of alternatively spliced isoforms of FGF13 expressed in the nervous system, epithelial tissues and tumours, suggesting humanspecific changes in isoform regulation (Supplementary Fig. 24).

\section{Discussion}

Comparative analysis of 29 mammalian genomes reveals a highresolution map of $>3.5$ million constrained elements that encompass $\sim 4 \%$ of the human genome and suggest potential functional classes for $\sim 60 \%$ of the constrained bases; the remaining $40 \%$ show no overlap and remain uncharacterized. We report previously undetected exons and overlapping functional elements within protein-coding sequence, new classes of RNA structures, promoter conservation profiles and predicted targets of transcriptional regulators. We also provide evidence of evolutionary innovation, including codon-specific positive selection, mobile element exaptation and accelerated evolution in the primate and human lineages.

By focusing our comparison on only eutherian mammals, we discover functional elements relevant to this clade, including recent eutherian innovations. This is especially important for discovering regulatory elements, which can be subject to rapid turnover ${ }^{47}$. Indeed, a previous comparison indicated that only $80 \%$ of 50 -bp non-coding elements are shared with opossum ${ }^{48}$, and the current 12-bp analysis shows $\sim 64 \%$ of non-coding elements shared with opossum, and only $6 \%$ with stickleback fish. Many eutherian elements are thus probably missing from previous maps of vertebrate constraint ${ }^{17}$

Sequencing of additional species should enable discovery of lineagespecific elements within mammalian clades, and provide increased resolution for shared mammalian constraint. We estimate that 100 200 eutherian mammals (15-25 neutral substitutions per site) will enable single-nucleotide resolution. The majority of this branch length is present within the Laurasiatherian and Euarchontoglire branches, which also contain multiple model organisms. These are ideal next targets for sequencing as part of the Genome $10 \mathrm{~K}$ effort ${ }^{49}$, aiming to sequence 10,000 vertebrate species. Within the primate clade, a branch length of $\sim 1.5$ could probably be achieved, enabling primate-specific selection studies, albeit at lower resolution. Lastly, human-specific selection should be detectable by combining data across genomic regions and by comparing thousands of humans ${ }^{50}$.

The constrained elements reported here can be used to prioritize disease-associated variants for subsequent study, providing a powerful lens for elucidating functional elements in the human genome complementary to ongoing large-scale experimental endeavours such as ENCODE and Roadmap Epigenomics. Experimental studies require prior knowledge of the biochemical activity sought and reveal regions active in specific cell types and conditions. Comparative approaches provide an unbiased catalogue of shared functional regions independent of biochemical activity or condition, and thus can capture experimentally intractable or rare activity patterns. With increasing branch length, they can provide information on ancestral and recent selective pressures across mammalian clades and within the human population. Ultimately, the combination of disease genetics, comparative and population genomics and biochemical studies have important implications for understanding human biology, health and disease.

\section{METHODS SUMMARY}

A full description of materials and methods, including sample selection and sequencing strategy, assembly strategies and results, error estimation and correction, alignment details, estimation of genome portion under constraint, detection of constrained elements, mammalian constraint versus human polymorphism, protein coding genes, detection of stop codon readthrough and synonymous constraint elements, RNA structure detection, patterns of promoter constraint, regulatory motif discovery, correlation with chromatin state information, overall accounting of constraint elements, comparison with disease-associated variants, detection of codon-specific positive selection, exaptation of ancestral repeat elements, and human and primate accelerated regions is available in Supplementary Information. All animal experiments were approved by the MIT Committee for Animal Care.

Received 25 January; accepted 5 September 2011.

Published online 12 October 2011.

1. Lander, E. S. et al. Initial sequencing and analysis of the human genome. Nature 409, 860-921 (2001)

2. Waterston, R. H. et al. Initial sequencing and comparative analysis of the mouse genome. Nature 420, 520-562 (2002)

3. Gibbs, R. A. et al. Genome sequence of the Brown Norway rat yields insights into mammalian evolution. Nature 428, 493-521 (2004).

4. Lindblad-Toh, K. et al. Genome sequence, comparative analysis and haplotype structure of the domestic dog. Nature 438, 803-819 (2005).

5. Altshuler, D., Daly, M. J. \& Lander, E. S. Genetic mapping in human disease. Science 322, 881-888 (2008)

6. Bejerano, G. et al. Ultraconserved elements in the human genome. Science $\mathbf{3 0 4}$ 1321-1325 (2004)

7. Kellis, M., Patterson, N., Endrizzi, M., Birren, B. \& Lander, E. S. Sequencing and comparison of yeast species to identify genes and regulatory elements. Nature 423, 241-254 (2003)

8. Stark, A. et al. Discovery of functional elements in 12 Drosophila genomes using evolutionary signatures. Nature 450, 219-232 (2007).

9. Cooper, G. M., Brudno, M., Green, E. D., Batzoglou, S. \& Sidow, A. Quantitative estimates of sequence divergence for comparative analyses of mammalian genomes. Genome Res. 13, 813-820 (2003)

10. Gnerre, S., Lander, E. S., Lindblad-Toh, K. \& Jaffe, D. B. Assisted assembly: how to improve a de novo genome assembly by using related species. Genome Biol. 10, R88 (2009).

11. Hubisz, M. J., Lin, M. F., Kellis, M. \& Siepel, A. Error and error mitigation in lowcoverage genome assemblies. PLOS ONE 14, e17034 (2011).

12. Thomas, J. W. et al. Comparative analyses of multi-species sequences from targeted genomic regions. Nature 424, 788-793 (2003).

13. Garber, M. et al. Identifying novel constrained elements by exploiting biased substitution patterns. Bioinformatics 25, i54-i62 (2009).

14. Chiaromonte, F.etal. The share of human genomic DNA under selection estimated from human-mouse genomic alignments. Cold Spring Harb. Symp. Quant. Biol. 68, 245-254 (2003).

15. Davydov, E. V. et al. Identifying a high fraction of the human genome to be under selective constraint using GERP++. PLOS Comput. Biol. 6, e1001025 (2010).

16. Meader, S., Ponting, C. P. \& Lunter, G. Massive turnover of functional sequence in human and other mammalian genomes. Genome Res. 20, 1335-1343 (2010).

17. Siepel, A. et al. Evolutionarily conserved elements in vertebrate, insect, worm, and yeast genomes. Genome Res. 15, 1034-1050 (2005).

18. Drake, J. A et al. Conserved noncoding sequences are selectively constrained and not mutation cold spots. Nature Genet. 38, 223-227 (2006).

19. Keinan, A., Mullikin, J. C., Patterson, N. \& Reich, D. Measurement of the human allele frequency spectrum demonstrates greater genetic drift in East Asians than in Europeans. Nature Genet. 39, 1251-1255 (2007).

20. Clamp, M. et al. Distinguishing protein-coding and noncoding genes in the human genome. Proc. Natl Acad. Sci. USA 104, 19428-19433 (2007).

21. Xie, X. et al. Systematic discovery of regulatory motifs in human promoters and 3' UTRs by comparison of several mammals. Nature 434, 338-345 (2005).

22. Wang, E. T. et al. Alternative isoform regulation in human tissue transcriptomes. Nature 456, 470-476 (2008).

23. Siepel, A. et al. Targeted discovery of novel human exons by comparative genomics. Genome Res. 17, 1763-1773 (2007).

24. Pruitt, K. D. et al. The consensus coding sequence (CCDS) project: identifying a common protein-coding gene set for the human and mouse genomes. Genome Res. 19, 1316-1323 (2009).

25. Lin, M. F., Jungreis, I. \& Kellis, M. PhyloCSF: a comparative genomics method to distinguish protein-coding and non-coding regions. Bioinformatics $27, \mathrm{i} 275-\mathrm{i} 282$ (2010).

26. Guttman, M. et al. Ab initio reconstruction of cell type-specific transcriptomes in mouse reveals the conserved multi-exonic structure of lincRNAs. Nature Biotechnol. 28, 503-510 (2010). 
27. Harrow, J. etal. GENCODE: producing a reference annotation for ENCODE. Genome Biol. 7 (suppl. 1), 1-9 (2006).

28. Lin, M. F. et al. Revisiting the protein-coding gene catalog of Drosophila melanogaster using 12 fly genomes. Genome Res. 17, 1823-1836 (2007).

29. Jungreis, I. et al. Evidence of abundant stop codon readthrough in Drosophila and other metazoa. Genome Res. doi:10.1101/gr.119974.110 (in the press).

30. Washietl, S., Hofacker, I. L. \& Stadler, P. F. Fast and reliable prediction of noncoding RNAs. Proc. Natl Acad. Sci. USA 102, 2454-2459 (2005).

31. Lin, M. F. et al. Locating protein-coding sequences under selection for additional, overlapping functions in 29 mammalian genomes. Genome Res. doi:10.1101/ gr.108753.110 (in the press).

32. Tumpel, S., Cambronero, F., Sims, C., Krumlauf, R. \& Wiedemann, L. M. A regulatory module embedded in the coding region of Hoxa2 controls expression in rhombomere 2. Proc. Natl Acad. Sci. USA 105, 20077-20082 (2008).

33. Lampe, $X$. et al. An ultraconserved Hox-Pbx responsive element resides in the coding sequence of Hoxa2 and is active in rhombomere 4. Nucleic Acids Res. 36 , 3214-3225 (2008)

34. Pedersen, J. S. et al. Identification and classification of conserved RNA secondary structures in the human genome. PLOS Comput. Biol. 2, e33 (2006).

35. Lee, J. T. Lessons from X-chromosome inactivation: Iong ncRNA as guides and tethers to the epigenome. Genes Dev. 23, 1831-1842 (2009).

36. Maenner, S. et al. 2-D structure of the A region of Xist RNA and its implication for PRC2 association. PLoS Biol. 8, e1000276 (2010).

37. Parker, B. J. et al. New families of human regulatory RNA structures identified by comparative analysis of vertebrate genomes. Genome Res. doi:10.1101/ gr.112516.110 (in the press).

38. Martinez-Chantar, M. L. et al. L-methionine availability regulates expression of the methionine adenosyltransferase $2 \mathrm{~A}$ gene in human hepatocarcinoma cells: role of S-adenosylmethionine. J. Biol. Chem. 278, 19885-19890 (2003).

39. Baek, D., Davis, C.. Ewing, B., Gordon, D. \& Green, P. Characterization and predictive discovery of evolutionarily conserved mammalian alternative promoters. Genome Res. 17, 145-155 (2007).

40. Kheradpour, P., Stark, A., Roy, S. \& Kellis, M. Reliable prediction of regulator targets using 12 Drosophila genomes. Genome Res. 17, 1919-1931 (2007).

41. Ernst, J. \& Kellis, M. Discovery and characterization of chromatin states for systematic annotation of the human genome. Nature Biotechnol. 28, 817-825 (2010).

42. Ernst, J. et al. Mapping and analysis of chromatin state dynamics in nine human cell types. Nature 473, 43-49 (2011).

43. Pillas, D. etal. Genome-wide association study reveals multiple loci associated with primary tooth development during infancy. PLoS Genet. 6, e1000856 (2010).

44. Lowe, C. B., Bejerano, G. \& Haussler, D. Thousands of human mobile element fragments undergo strong purifying selection near developmental genes. Proc. Natl Acad. Sci. USA 104, 8005-8010 (2007).

45. Prabhakar, S. et al. Human-specific gain of function in a developmental enhancer Science 321, 1346-1350 (2008).

46. Pollard, K. S. et al. Forces shaping the fastest evolving regions in the human genome. PLoS Genet. 2, e168 (2006).

47. Schmidt, D. et al. Five-vertebrate ChIP-seq reveals the evolutionary dynamics of transcription factor binding. Science 328, 1036-1040 (2010).

48. Mikkelsen, T. S. et al. Genome of the marsupial Monodelphis domestica reveals innovation in non-coding sequences. Nature 447, 167-177 (2007).

49. Genome 10K Community Of Scientists. Genome 10K: a proposal to obtain wholegenome sequence for 10,000 vertebrate species. J. Hered. 100, 659-674 (2009).

50. A map of human genome variation from population-scale sequencing. Nature 467, 1061-1073 (2010).

Supplementary Information is linked to the online version of the paper at www.nature.com/nature.

Acknowledgements We thank O. Ryder, E. Fuchs, D. Haring, A. Walsh, D. Duffield S. Wong, T. Alvarado, J. Boylan, S. Combes, P. de Jong, J. Allman, J. Patton, D. McMullen, D. Hafner, D. Miller, T. Kunz, G. Hewitt, J. Searle, H. Künzle and D. Williams for providing organismal material. We thank L. Gaffney for help with figures. This work was supported by the National Human Genome Research Institute (NHGRI), including grant U54 HG003273 (R.A.G.), National Institute for General Medicine (NIGMS) grant no.
GM82901 (Pollard laboratory) and the European Science Foundation (EURYI award to K.L.-T.), NSF National Science Foundation (NSF) postdoctoral fellowship award 0905968 (J.E.), National Science Foundation CAREER 0644282 and NIH R01 HG004037 and the Sloan Foundation (M.K.), and an Erwin Schrödinger Fellowship of the Austrian Fonds zur Förderung der Wissenschaftlichen Forschung (S.W.), the Gates Cambridge Trust (G.J.), Novo Nordisk Foundation (B.J.P. and J.W.); a Statistics Network Fellowship, Department of Mathematical Sciences, University of Copenhagen (B.J.P.); the David and Lucile Packard Foundation (A.S.); the Danish Council for Independent Research Medical Sciences (J.S.P.); The Lundbeck Foundation (J.S.P.)

Author Contributions K.L-T.E.S.L and M.K. led the project and oversaw the analysis. K.L.-T. M.C., J.Ch. E.H.M., E.D.G. and E.S.L. planned the project. K.L.-T., F.D.P., M.L., E.S.L., K.C.W., C.L.K., D.M.M., R.A.G., W.C.W., E.R.M., G.M.W. and R.K.W. oversaw or significantly contributed to data generation. S.G. assembled the $2 \times$ genomes. Major contributions to analysis were made by M.Ga., O.Z. and M.C. to evaluate measures and patterns of evolutionary selection, M.F.L. to evaluate protein-coding potential and translational readthrough, B.J.P., S.W. to analyse RNA structures and families, P.K. on regulatory motifs and motif instances, J.E. on chromatin states, G.J. on codon-specific positive selection, E.M. on promoter motif, L.D.W. on GWAS overlap with conserved elements, C.B.L. on exaptation and A.K.H., K.S.P. on HARs and PARs. J.A., K.B., H.C., J.Cu., S.F., P.F., M.Gu., M.J.H., D.B.J., I.J., W.J.K., D.K., A.L.M., T.M., I.M., B.J.R., M.D.R., J.R., A.St., A.J.V., J.W, X.X., M.C.Z., E.B., E.H.M., J.H., D.H., A.Si., N.G. and J.S.P. performed or oversaw various analyses. K.L.-T., E.S.L. and M.K. wrote the paper with input from the other authors.

Author Information A complete set of data files can be downloaded from the Broad website (https://www.broadinstitute.org/scientific-community/science/projects/ mammals-models/29-mammals-project-supplementary-info) or viewed using the UCSC Genome Browser (http://genomewiki.cse.ucsc.edu/index.php/29mammals) or the Broad Institute Integrative Genome Viewer (http://www.broadinstitute.org/igv/ projects/29mammals). NCBI accession numbers for all newly sequenced genomes can be found in Supplementary Table 1. Reprints and permissions information is available at www.nature.com/reprints. This paper is distributed under the terms of the Creative Commons Attribution-Non-Commercial-Share Alike licence, and is freely available to all readers at www.nature.com/nature. The authors declare no competing financial interests. Readers are welcome to comment on the online version of this article at www.nature.com/nature. Correspondence and requests for materials should be addressed to to K.L.-T. (kersli@broadinstitute.org), E.S.L. (lander@broadinstitute.org) or M.K. (manoli@mit.edu).

\section{Broad Institute Sequencing Platform and Whole Genome Assembly Team}

Jen Baldwin ${ }^{1}$, Toby Bloom ${ }^{1}$, Chee Whye Chin $^{1}$, Dave Heiman ${ }^{1}$, Robert Nicol ${ }^{1}$, Chad Nusbaum ${ }^{1}$, Sarah Young ${ }^{1} \&$ Jane Wilkinson ${ }^{1}$

\section{Baylor College of Medicine Human Genome Sequencing Center Sequencing Team}

Andrew Cree ${ }^{2}$, Huyen H. Dihn ${ }^{2}$, Gerald Fowler ${ }^{2}$, Shalili Jhangiani ${ }^{2}$, Vandita Joshi ${ }^{2}$ Sandra Lee ${ }^{2}$, Lora R. Lewis ${ }^{2}$, Lynne V. Nazareth ${ }^{2}$, Geoffrey Okwuonu² \& Jireh Santibanez

\section{Genome Institute at Washington University}

Kim Delehaunty ${ }^{3}$, David Dooling ${ }^{3}$, Catrina Fronik ${ }^{3}$, Lucinda Fulton ${ }^{3}$, Bob Fulton ${ }^{3}$, Tina Graves $^{3}$, Patrick Minx ${ }^{3}$ \& Erica Sodergren ${ }^{3,4}$

${ }^{1}$ Broad Institute of Harvard and Massachusetts Institute of Technology (MIT), 320 Charles Street, Cambridge, Massachusetts 02142, USA. ${ }^{2}$ Human Genome Sequencing Center, Baylor College of Medicine, One Baylor Plaza, Houston, TX77030, USA. ${ }^{3}$ Genome Institute at Washington University, Washington University School of Medicine, 4444 Forest Park Blvd, Saint Louis, Missouri 63108, USA. ${ }^{4}$ Research Institute of Molecular Pathology (IMP), A-1030 Vienna, Austria. 